\title{
Hashimoto's Thyroiditis Is a Risk Factor for Thyroid Cancer
}

\section{Stephanie A. Fish}

Department of Endocrinology, Memorial Sloan Kettering Cancer Center, New York, New York, U.S.A.

Review of: Silva de Morais N, Stuart J, Guan H, Wang Z, Cibas ES, Frates MC, Benson CB, Cho NL, Nehs MA, Alexander CA, Marqusee E, Kim MI, Lorch JH, Barletta JA, Angell TE, Alexander EK 2019 The impact of Hashimoto thyroiditis on thyroid nodule cytology and risk of thyroid Cancer. J Endocr Soc 3:791-800. PMID: 30963137

\section{SUMMARY}

\section{Background}

Hashimoto's thyroiditis (HT), a common autoimmune disease, is the most frequent cause of hypothyroidism, affecting 2 to $15 \%$ of the population (1). Histologically, HT is characterized by diffuse lymphocytic infiltration of the thyroid gland (2). It has been hypothesized that HT, a chronic thyroid inflammatory disease accompanied by positive serum thyroid peroxidase antibody (TPOAb) titers, is associated with an increased risk of thyroid cancer. However, previous studies have not been able to prove this conclusively. In this study, the authors used a large prospectively tracked database of patients evaluated for thyroid nodules to determine whether there is an association between HT and thyroid cancer, as well as to determine the predictive capability of HT on diagnostic thyroid nodule evaluation and thyroid cytology results.

\section{Methods}

This is a prospective cohort study of 10,054 consecutive adult patients with thyroid nodules $>1 \mathrm{~cm}$ who were evaluated between 1995 and 2017 at the Brigham and Women's Hospital Thyroid Nodule Clinic (3). All patients underwent sonographic and clinical assessment. From the ultrasound report, HT was considered present if the background parenchyma was diffusely heterogeneous or the presence of HT was reported (4). Serum TPOAb levels were measured ( $n=2551$ patients) when the serum TSH level was elevated or at the discretion of the clinician. The TPOAb test was considered positive when the antibody level was above the reference range.

Thyroid nodules were treated according to the clinical guidelines at the time of evaluation. Fine-needle aspiration (FNA) was performed with ultrasound guidance when indicated. FNA specimens were processed using ThinPrep liquid-based cytology preparation. Thyroid cytology was reported as nondiagnostic, negative for malignant cells, atypia of undetermined significance, suspicious for follicular or Hürthle-cell neoplasm, suspicious for malignancy, or malignant. Surgery was recommended if cytologic findings were suspicious for malignancy or malignant. When thyroidectomy was performed $(n=$ 3186 patients), histopathologic data were obtained to document malignant or benign disease as well as histologic evidence of HT.

The investigators used a very general definition of $\mathrm{HT}$ that included an elevated TPOAb level and/or findings of diffuse heterogeneity on ultrasound and/ or diffuse lymphocytic thyroiditis on histopathology. 

Factor for Thyroid Cancer

\section{Results}

The final cohort included 9851 patients with 21,397 nodules. The population was predominantly female (83.9\%) with a mean age of 52.2 years. A total of $14,063(66 \%)$ of the nodules were evaluated with FNA. HT was diagnosed in 2651 patients (27\%) with a total of 3895 nodules.

The proportion of nodules with indeterminate and malignant cytology was higher in the HT group as compared with the non-HT group. Indeterminate cytology was found in $20.6 \%$ of HT patients with nodules, while it was identified in only $17.1 \%$ of non-HT patients $(P<0.01)$. An increase in malignant cytology was also identified in HT patients. Cancer was diagnosed on histopathology more frequently in the HT patients as compared with the non-HT patients $(23.3 \%$ vs. $15.9 \%, P<0.01)$. There were no significant differences in pathologic characteristics or markers of aggressiveness between the two groups.

Of note, 13 cases of thyroid lymphoma were identified (9 in patients without HT and 4 in patients with $\mathrm{HT})$.

\section{Conclusions}

In patients with thyroid nodules, HT is associated with a higher rate of indeterminate cytology and a higher incidence of thyroid malignancy. Assessment of HT is an important part of a thyroid nodule evaluation.

\section{COMMENTARY}

This large prospective cohort study looked at the association between HT and thyroid malignancy. The investigators found an increased rate of indeterminate cytology, as well as an increased incidence of thyroid cancer among patients with HT.

For manyyears, there have been questions regarding the association between thyroid inflammation from HT and an increased risk of thyroid cancer. The results of previous studies have been inconclusive. In this study, the investigators evaluated a very large cohort of patients prospectively. The patient population was selected based on the presence of a clinically significant thyroid nodule. Thus, it does not represent the general population. But, based on these results, it appears that a nodule in a patient with $\mathrm{HT}$ is more likely to be thyroid cancer. This is a clinically significant finding as both HT and thyroid nodules are so common. It may be necessary to consider thyroid ultrasound in all HT patients to evaluate for the presence of thyroid nodules.
The investigators used a very broad definition of $\mathrm{HT}$, including all patients with positive TPOAb levels and/or diffuse heterogeneity on ultrasound and/ or diffuse lymphocytic thyroiditis on histopathology. This is a reasonable approach to ensure that all patients with HT are categorized correctly. However, they did not mention whether all the HT patients who underwent thyroid surgery had evidence of HT on histopathology. Diffuse lymphocytic thyroiditis on histopathology is the gold standard for the diagnosis of HT, and it would be difficult to include a patient in the HT group if they did not have this finding at surgery.

It is not surprising that HT patients have a higher rate of indeterminate cytology. The inflammation associated with HT can cause reactive atypia in the thyroid follicular cells that mimics the findings in papillary thyroid cancer, such as increased nuclear size and nuclear contour irregularities and grooves, that will result in an indeterminate diagnosis for FNA samples 
(5). Thus, this observation is expected. It would be interesting to look at the proportion of the atypical nodules that are malignant on histopathology to see whether HT is associated with an increased risk of malignancy in this subgroup of nodules.

Cancer was found in patients with both solitary and multiple thyroid nodules. This supports the hypothesis that inflammation imparts a field effect throughout the thyroid gland. While the investigators found an increased risk of malignancy associated with $\mathrm{HT}$, they did not find that the tumors were more aggressive (3). It appears that the inflammatory effect does not affect the pathologic features of the cancer. In the future, it may be worthwhile to study the genetic mutations associated with thyroid cancer in HT patients versus non-HT patients.

This large prospective cohort study of thyroid nodule patients showed an increased risk of indeterminate cytology and thyroid malignancy associated with HT. This is a clinically significant finding, as it can help to determine the best approach to evaluating these patients. It would be interesting to confirm these findings in a more general population and to determine the pathophysiology of this association.

\section{References}

1. McLeod DS, Cooper DS 2012 The incidence and prevalence of thyroid autoimmunity. Endocrine 42:252-265.

2. Caturegli P, De Remigis A, Chuang K, Dembele M, Iwama A, Iwama S 2013 Hashimoto's thyroiditis: celebrating the centennial through the lens of the Johns Hopkins hospital surgical pathology records. Thyroid 23:142-150.

3. Silva de Morais N, Stuart J, Guan H, Wang Z, Cibas ES, Frates MC, Benson CB, Cho NL, Nehs MA, Alexander CA, et al. 2019 The impact of Hashimoto thyroiditis on thyroid nodule cytology and risk of thyroid Cancer. J Endocr Soc 3:791-800.
4. Patel S, Giampoli E, Oppenheimer D, Montoya S, Rupasov A, Dogra V 2018 Sonographic features of diffuse Hashimoto thyroiditis: determining sensitivity of features and predictors of malignancy. Am J Sonogr 1:1-7.

5. Harvey AM, Truong LD, Mody DR 2012 Diagnostic pitfalls of Hashimoto's/lymphocytic thyroiditis on fine-needle aspirations and strategies to avoid overdiagnosis. Acta Cytol 56:352-360. 